\title{
Single Nucleotide Polymorphism
} Analysis Indicates Genetic Distinction and Reduced Diversity of Swine-Associated Methicillin Resistant Staphylococcus aureus (MRSA) ST5 Isolates Compared to Clinical MRSA ST5 Isolates

\section{OPEN ACCESS}

Edited by:

Eric Altermann,

AgResearch, New Zealand

Reviewed by:

Santiago Castillo Ramírez,

Universidad Nacional Autónoma de México, Mexico

Francisco Rodriguez-Valera, Universidad Miguel Hernández de Elche, Spain

*Correspondence:

Tracy L. Nicholson

tracy.nicholson@ars.usda.gov

Specialty section: This article was submitted to

Evolutionary and Genomic Microbiology,

a section of the journal

Frontiers in Microbiology

Received: 03 May 2018

Accepted: 14 August 2018 Published: 11 September 2018

Citation:

Hau SJ, Allué-Guardia A, Rusconi B,

Haan JS, Davies PR, Frana TS, Eppinger M and Nicholson TL (2018)

Single Nucleotide Polymorphism Analysis Indicates Genetic Distinction and Reduced Diversity of Swine-Associated Methicillin Resistant Staphylococcus aureus (MRSA) ST5 Isolates Compared to Clinical MRSA ST5 Isolates.

Front. Microbiol. 9:2078. doi: 10.3389/fmicb.2018.02078

\begin{abstract}
Samantha J. Hau1, Anna Allué-Guardia ${ }^{2,3}$, Brigida Rusconi2,3, Jisun S. Haan4, Peter R. Davies ${ }^{4}$, Timothy S. Frana1, Mark Eppinger ${ }^{2,3}$ and Tracy L. Nicholson ${ }^{5 *}$

'Department of Veterinary Diagnostic and Production Animal Medicine, College of Veterinary Medicine, lowa State University, Ames, IA, United States, ${ }^{2}$ South Texas Center for Emerging Infectious Diseases, University of Texas at San Antonio, San Antonio, TX, United States, ${ }^{3}$ Department of Biology, University of Texas at San Antonio, San Antonio, TX. United States, ${ }^{4}$ Department of Veterinary Population Medicine, College of Veterinary Medicine, University of Minnesota, Saint Paul, MN, United States, ${ }^{5}$ National Animal Disease Center, Agricultural Research Service, United States Department of Agriculture, Ames, IA, United States
\end{abstract}

Livestock associated methicillin resistant S. aureus (LA-MRSA) are lineages adapted to livestock species. LA-MRSA can be transmitted to humans and public health concerns exist because livestock may be the largest MRSA reservoir outside of hospital settings. Although the predominant European (ST398) and Asian (ST9) lineages of LAMRSA are considered livestock adapted, North American swine also harbor ST5, a globally disseminated and highly pathogenic lineage. This study applied whole genome sequencing and single nucleotide polymorphism (SNP) typing to compare the population structure and genetic relatedness between swine associated and human clinical MRSA ST5 isolates. The established high-resolution phylogenomic framework revealed that LA-MRSA and human clinical MRSA ST5 are genetically distinct. LA-MRSA isolates were found to be clonal within farms, while greater genome diversity was observed among sampled clinical MRSA ST5. Analysis of the accessory genome demonstrated that LA-MRSA ST5 isolates and clinical MRSA ST5 isolates harbor different AMR genes and virulence factors, consistent with the SNP analysis. Collectively, our data indicate LA-MRSA and clinical MRSA ST5 isolates are distinct and the swine reservoir is likely of minimal significance as a source of clinical MRSA ST5 infections.

Keywords: LA-MRSA, Staphylococcus aureus, whole genome sequence (WGS), single nucleotide polymorphism
(SNP) typing, phylogenetic analysis, swine, mobile genetic elements, agriculture

\section{INTRODUCTION}

Staphylococcus aureus is a commensal organism found in the nasopharynx and on the skin of humans and other mammals. It can also cause infections in these hosts and cause a range of diseases from mild skin and soft tissue infections to severe systemic infections. The treatment of $S$. aureus infections is hampered by the development of antimicrobial resistance (AMR) in these isolates, 
such as the acquisition of the SCCmec element, which confers methicillin resistance. These isolates are designated as methicillin resistant $S$. aureus (MRSA) and have become a significant burden for the health care system (Klevens et al., 2007; DeLeo and Chambers, 2009).

Methicillin resistant $S$. aureus isolates are classified based on epidemiologic characteristics into hospital acquired MRSA (HAMRSA), community acquired MRSA (CA-MRSA), and livestock associated MRSA (LA-MRSA) (Stefani et al., 2012). These subsets are defined by the source of the isolate and the isolates within each subset tend to share genotypic and phenotypic characteristics, such as degree of AMR and virulence factors. Specific lineages or sequence types (STs) tend to predominate within each group, although there are regional variations. For example, in the United States, ST5 and ST8 are major HA-MRSA and CA-MRSA clones, respectively (Bal et al., 2016).

Livestock associated MRSA became a significant public health concern in 2005, when the first report linked MRSA ST398 to swine production facilities (Voss et al., 2005). Further investigations indicated swine may serve as the largest reservoir for MRSA outside of hospital settings and motivated considerable research into the potential health risks associated with LAMRSA (Schijffelen et al., 2010). Subsequent research showed the most prevalent lineage of LA-MRSA varied based on geographic location. In European swine populations, ST398 is the most common lineage (Andreoletti et al., 2009), while the ST9 lineage predominates in swine in most Asian countries (Wagenaar et al., 2009). In the United States, the swine population was found to harbor more diverse STs with isolates of the ST398, ST9, and ST5 lineages (Molla et al., 2012; Frana et al., 2013; Sun et al., 2015). The presence of MRSA ST5 isolates in United States swine herds raised additional public health concerns because the ST5 lineage, unlike the ST398 and ST9 lineages, is not considered livestock adapted but is a highly successful and globally disseminated MRSA lineage in and out of hospital settings (Monecke et al., 2011; Fitzgerald, 2012; Pantosti, 2012). The widespread success of the ST5 lineage has been attributed to its capacity to acquire mobile genetic elements (MGEs) that harbor virulence factors or AMR genes (Monecke et al., 2011).

Phylogenetic studies employ whole genome sequencing (WGS) technology and data analysis to better understand the epidemiology, origin, and evolution of bacteria (Price et al., 2012; Uhlemann et al., 2012; Eppinger et al., 2014; Sahibzada et al., 2017). In the case of LA-MRSA, these techniques have been used to assess the relatedness of isolates from the ST398 lineage. LAMRSA ST398 isolates clustered separately from human ST398 isolates and are suspected to have evolved from an ancestral methicillin susceptible ST398 clade in humans (Price et al., 2012). Similar investigations into the CC97 lineage indicated that isolates causing clinical disease in humans comprise a sub-clade of the LA-MRSA isolates that may have developed an increased capacity for infecting and causing disease in humans (Spoor et al., 2013). WGS analysis can be used to assess isolate relatedness or determine genetic characteristics that define subsets of isolates, including information about the MGEs harboring virulence factors or AMR genes. Through single nucleotide polymorphism (SNP) discovery within the core genome, the accuracy and resolution power is available to determine phylogenetic relationships and distinguish isolates within highly homogenous lineages, which provides insight in epidemiological investigations (Eppinger et al., 2011, 2014; Rusconi et al., 2016; Sahibzada et al., 2017).

Although previous reports have examined the genetic diversity and relatedness of MRSA isolates from livestock species and humans within the ST398 and CC97 lineages (Price et al., 2012; Spoor et al., 2013), to date there are no reports using WGS data to evaluate the genetic diversity of MRSA ST5 isolates obtained from swine and human sources. In this study, we used SNP analysis of the core genome to evaluate the population structure and genetic diversity of LA-MRSA ST5 isolates from a variety of swine associated sources and clinical MRSA ST5 isolates from humans with no swine contact to investigate the potential for LA-MRSA ST5 isolates to act as a source for human infections or as a source for MGEs harboring virulence factors or AMR.

\section{MATERIALS AND METHODS}

\section{Isolate Acquisition}

Swine associated LA-MRSA ST5 isolates were obtained from samples collected while visiting pork farms in the Midwest United States as part of field case investigations completed by Iowa State University (ISU) (Frana et al., 2013). Sources for these isolates were swine nasal swabs (38 isolates), the environment within swine facilities (25 isolates), and humans with short-term contact with swine (9 isolates) (Frana et al., 2013). Isolates from healthy practicing swine veterinarians were provided by the University of Minnesota (UMN). The isolates $(n=9)$ were acquired from swine veterinarians who participated in a longitudinal study focusing on $S$. aureus. Veterinarians resided in areas of intensive swine production in the Midwest and Southeast United States and had regular (i.e., greater than twice per week) professional contact with pigs (Sun et al., 2017). MRSA ST5 isolates from humans with no swine contact were obtained from medical centers associated with the University of California Irvine (UCI) $(n=64)$ (Hudson et al., 2013) and the University of California, San Francisco (UCSF) $(n=8)$. Isolates were characterized for MLST and Spa typed prior to acquisition (Frana et al., 2013; Hudson et al., 2013; Sun et al., 2017). Specific isolate information can be found in Supplementary Table S1. All isolates were either obtained from samples collected as part of previous studies and obtained through written consistent (Frana et al., 2013; Hudson et al., 2013; Sun et al., 2017) or were obtained from samples submitted as part of field case investigations and did not require Institutional Animal Care and Use Committee (IACUC) approval.

\section{Whole Genome Sequencing}

Draft genomes were generated as previously described (Hau et al., 2017a,b,c,d,e,f). Briefly, total genomic DNA was extracted from isolates grown in Trypticase Soy Broth (BD Biosciences, Sparks, MD, United States) using a High Pure PCR Template Preparation Kit (Roche Applied Science, Indianapolis, IN, United States). The Nextera XT DNA sample preparation and index kit (Illumina, 
San Diego, CA, United States) was used to generate pairedend DNA libraries with 250-bp read length that were sequenced using the MiSeq v2 500 Cycle reagent kit on the Illumina MiSeq platform (Illumina, San Diego, CA, United States). Sequence reads were assembled with MIRA v. 4.0.2 ${ }^{1}$ and annotated using the NCBI Prokaryotic Genome Annotation Pipeline ${ }^{2}$. Draft genomes and sequence reads have been submitted to GenBank and the Sequence Read Archive (SRA). Accession numbers are provided in Supplementary Table $\mathbf{S 1}$.

\section{Core Genome SNP Discovery}

For reference based SNP discovery we used a custom developed pipeline implemented on Galaxy (Goecks et al., 2010), that was successfully applied for the high resolution genomic epidemiology profiling of various microbial human pathogens (Eppinger et al., 2011, 2014; Rusconi et al., 2016). Strategies and phylogenetic principles have been described in detail in Rusconi et al., 2016. The MRSA core genome in this study is defined in the samples as the set of genic and intragenic regions that are present in all genomes, not repeated, and do not contain MGEs (which evolve at different rates and are not indicative of evolutionary relationships), such as phages, IS elements, genomic islands or plasmids. These excluded regions were determined for the reference genome Mu50 as follows: NUCmer was used to detect repeat regions by running the reference against itself (Delcher et al., 2003), integrated bacteriophages were identified using PHAST (Zhou et al., 2011), ISFinder was used for detection of insertion sequences (Siguier et al., 2006), and antibiotic resistance cassettes were detected with ResFinder (Zankari et al., 2012). The SNP discovery and verification pipelines are implemented on Galaxy, and contain the following modules: (i) SNP discovery and typing, (ii) SNP curation, (iii) SNP annotation, (iv) SNP distribution, and (v) SNP phylogeny.

\section{SNP Discovery and Typing}

Illumina reads of the 72 MRSA ST5 isolates from humans with no swine contact and 82 LA-MRSA ST5 were uploaded into Galaxy along with two ST5 representative closed genomes: a HA-MRSA isolate from Japan (Mu3) and a representative poultry-adapted MRSA ST5 isolate (ED98) (Hiramatsu et al., 1997; Lowder et al., 2009). For read-based SNP discovery, reads were aligned with Bowtie 2 to the reference genome Mu50 (Langmead and Salzberg, 2012). The resulting alignments were processed with Freebayes using the following threshold settings: mapping quality 30 , base quality 20, coverage 30, and allelic frequency 0.9 (Garrison and Marth, 2012). The two closed representative genomes, Mu3 and ED98, were analyzed using the contig-based workflow. Briefly, a panel of SNPs for each genome was obtained by aligning the genome against the reference strain, Mu50, using NUCmer. SNPs were called with delta-filter and show-snps distributed with the MUMmer package (Delcher et al., 2003).

\section{SNP Curation}

Several SNP curation strategies were used to correct for false positive calls (Eppinger et al., 2011, 2014). First, reads were

${ }^{1}$ http://mira-assembler.sourceforge.net/docs/DefinitiveGuideToMIRA.html

${ }^{2}$ https://www.ncbi.nlm.nih.gov/genome/annotation_prok/ mapped against the reference genome Mu50 and false positives identified by Freebayes with the settings described. If reads were unavailable (Mu3 and ED98), the post-assembly workflow generated a reference-based NUCmer alignment and extracted SNPs as described above with filtering of false SNPs. SNPs located within excluded regions (repeat regions, bacteriophages, resistance cassettes, and IS elements) were removed. SNPs were further curated by extracting the 40 nucleotides surrounding each predicted SNP in the reference genome and completing a nucleotide BLAST against the query genomes (Altschul et al., 1990). Finally, resulting alignments were parsed to remove SNP locations derived from ambiguous hits $(\geq 2)$, low alignment quality or misalignments, non-uniformly distributed regions, and InDels, as previously described (Myers et al., 2009; Eppinger et al., 2011, 2014). Also, multinucleotide insertions and deletions of polymorphic bases were not considered SNPs and were excluded.

\section{SNP Annotation}

The curated cataloged SNPs from each query genome were merged into a single SNP panel that reported the allele, genic/intergenic status, SNP position, and annotation (Uhlemann et al., 2012; Spoor et al., 2013; Rusconi et al., 2016). This SNP discovery and validation pipeline allows for rapid typing of strains of unknown provenance by interrogating the captured allelic states from established SNP panels (Eppinger et al., 2011).

\section{SNP Distribution}

From the distribution of SNPs along the Mu50 chromosome, potential mutational hotspots and genes under positive selection could be identified using custom scripts implemented on Galaxy (Giardine et al., 2005; Myers et al., 2009; Goecks et al., 2010).

\section{SNP Phylogeny}

The identified curated SNP panel was used for phylogenetic reconstruction by maximum parsimony with PAUP v4.0a146 with 100 bootstrap replicates (Wilgenbusch and Swofford, 2003). The SNP tree was visualized in Geneious (vR9) and the majority consensus tree was built in Mesquite (Kearse et al., 2012; Maddison and Maddison, 2017). Tree decorations were added using Evolview (He et al., 2016). Calculation of the consistency index for each SNP allowed for identification of parsimony informative SNPs and flag homoplastic SNPs, as described in our previous works (Eppinger et al., 2014; Rusconi et al., 2016). A Maximum Likelihood phylogenetic tree was additionally inferred using RAxML v8.2.11 with the GTR CAT model and 100 bootstrap replicates (Stamatakis, 2014). The consensus tree was built and visualized in Geneious (vR9) (Kearse et al., 2012).

\section{Mobile Genetic Element Analysis}

Mobile genetic elements harboring AMR genes and virulence factors were detected as described previously and verified using Geneious 9.0.5 (Hau et al., 2015, 2017g; Hau, 2017). AMR genes detected with ResFinder were verified through draft genome analysis (Hau, 2017). The SCCmec type was determined using PCR and confirmed in silico (Hau et al., 2017g). Immune evasion genes associated with the $\beta$-hemolysin converting bacteriophage 
were identified in these isolates using PCR and confirmed in silico (Hau et al., 2015).

\section{RESULTS}

\section{Isolate Provenance and Sequence Information}

Swine associated LA-MRSA ST5 isolates were obtained from nasal swabs of healthy pigs or humans, none of which exhibited signs of MRSA infection, or from swabs of the environment within swine facilities. Clinical MRSA ST5 isolates were obtained from patients with MRSA related disease at two urban, university affiliated hospitals in California where the likelihood that patients had contact with swine was considered negligible. Draft genomes confirmed the MLST data indicating all isolates were ST5.

Draft genome sequences were generated for the 81 LA-MRSA ST5 isolates and 72 MRSA ST5 isolates from humans with no swine contact (Supplementary Table S1). Genome statistics are summarized in Supplementary Table S1.

\section{Single Nucleotide Polymorphism Typing}

For reference based SNP discovery, genomes were aligned to the closed genome of reference strain Mu50, a vancomycin resistant HA-MRSA isolate from Japan (Hiramatsu et al., 1997).

TABLE 1 | Non-synonymous SNPs unique to LA-MRSA ST5 isolates or MRSA ST5 isolates from humans with no swine contact.

\begin{tabular}{|c|c|c|c|}
\hline $\begin{array}{l}\text { Number of swine } \\
\text { associated isolates }\end{array}$ & $\begin{array}{l}\text { Number of isolates } \\
\text { from humans with no } \\
\text { swine contact }\end{array}$ & $\begin{array}{c}\text { SNP } \\
\text { position }^{\text {a }}\end{array}$ & Gene product \\
\hline $82 / 82$ & $0 / 72$ & 160799 & $\begin{array}{l}2^{\prime}-3^{\prime}-\text { Cyclic- } \\
\text { nucleotide } \\
2^{\prime}- \\
\text { phosphodiesterase }\end{array}$ \\
\hline $82 / 82$ & $0 / 72$ & 292343 & $\begin{array}{l}\text { Sorbitol } \\
\text { dehydrogenase } \\
\text { homolog }\end{array}$ \\
\hline $82 / 82$ & $0 / 72$ & 806489 & Putative transporte \\
\hline $82 / 82$ & $0 / 72$ & 848820 & $\begin{array}{l}\text { Putative } \\
\text { P-loop-containing } \\
\text { kinase }\end{array}$ \\
\hline $82 / 82$ & $0 / 72$ & 1012841 & $\begin{array}{l}\text { Similar to } \\
\text { ATP-dependent } \\
\text { nuclease subunit A }\end{array}$ \\
\hline $82 / 82$ & $0 / 72$ & 1928498 & $\begin{array}{l}\text { O-Succinylbenzoic } \\
\text { acid-CoA ligase }\end{array}$ \\
\hline $82 / 82$ & $0 / 72$ & 2695325 & $\begin{array}{l}\text { Ferrous iron } \\
\text { transport protein B } \\
\text { homolog }\end{array}$ \\
\hline $82 / 82$ & $0 / 72$ & 2720180 & Regulatory protein \\
\hline 0/82 & $69 / 72$ & 192929 & $\begin{array}{l}\text { Hypothetical } \\
\text { protein }\end{array}$ \\
\hline $0 / 82$ & $69 / 72$ & 2277937 & $\begin{array}{l}\text { Conserved } \\
\text { hypothetical proteir }\end{array}$ \\
\hline
\end{tabular}

aSNP position indicates the location of the SNP in the reference genome of Mu50. ${ }^{b}$ Gene product represents the protein produced by the gene containing the SNP of interest.
The core genome was determined by excluding identified MGEs and repeats. Core genome SNP discovery identified 764 SNPs comprised of: 154 intergenic, 186 synonymous, and 424 nonsynonymous SNPs (Supplementary Tables S2, S3). Further evaluation of non-synonymous SNPs indicated that eight were shared by swine associated LA-MRSA ST5 isolates and not present in isolates from humans with no swine contact, as listed in Table 1. There were also two SNPs found only in MRSA ST5 isolates from humans with no swine contact (Table 1) that were specific to clinical isolates. The genes harboring the nonsynonymous SNPs distinct to each subset of isolates have not been implicated in virulence of $S$. aureus and are unlikely to contribute to the pathogenicity of these isolates. SNPs were distributed throughout the Mu50 reference genome and no mutational hotspots were observed (Figure 1).

\section{Phylogenetic Analysis}

A phylogenetic hypothesis was constructed from the identified core genome SNPs and rooted using the MRSA ST5 isolate Mu50. This tree depicts the evolutionary relationships between the 153 MRSA ST5 isolates and the reference isolates Mu3 and ED98 (Figure 2). The tree topology shows that swine associated LA-MRSA ST5 isolates cluster together and are separated from MRSA ST5 isolates from humans with no swine contact. These groups will be referred to as Clades I and II, representing MRSA ST5 isolates from humans with no swine contact and LA-MRSA ST5, respectively. A single MRSA ST5 isolate (UCSF14436) from a human with no swine contact was contained within Clade II. This isolate was the most distantly related of the Clade II isolates, harboring 24 unique/strain-specific SNPs. USCF14436 harbors a type IV SCCmec element and two AMR genes (mecA and blaZ), which is a strong indication this isolate is a CA-MRSA strain (Figure 3).

Methicillin resistant $S$. aureus ST5 isolates from humans with no swine contact (Clade I) are divided into subclades Ia and Ib. The SNP-level plasticity between MRSA ST5 isolates from humans with no swine contact is greater than that from LA-MRSA ST5 isolates. Despite the northern and southern geographic regions represented by the two groups of California isolates, the tree topology shows that UCSF isolates were found interspersed throughout Clade I and did not cluster by geographic location.

The swine associated isolates within Clade II formed four subclades, IIa-d. Each subclade represents a subset of isolates from a specific farm or farms; such that, Clade IIa represents isolates from Farm 10, Clade IIb represents isolates from Farm 24, Clade IIc represents isolates from Farm 46, and Clade IId represents isolates from Farms 38-42. Isolates originating from individual farms were genetically homogenous, possessing fewer than five SNPs (distinguishing them from other isolates from the same farm). These results suggest that LA-MRSA ST5 populations residing on farms are clonal and intermingling/transfer of isolates among farms or reintroduction of MRSA ST5 onto farms had likely not occurred. This was true for all swine associated isolates except those from Farms 3842 (Clade IId). The high degree of genetic relatedness of the isolates within Clade IId suggests these farms are likely from a 
BA000017

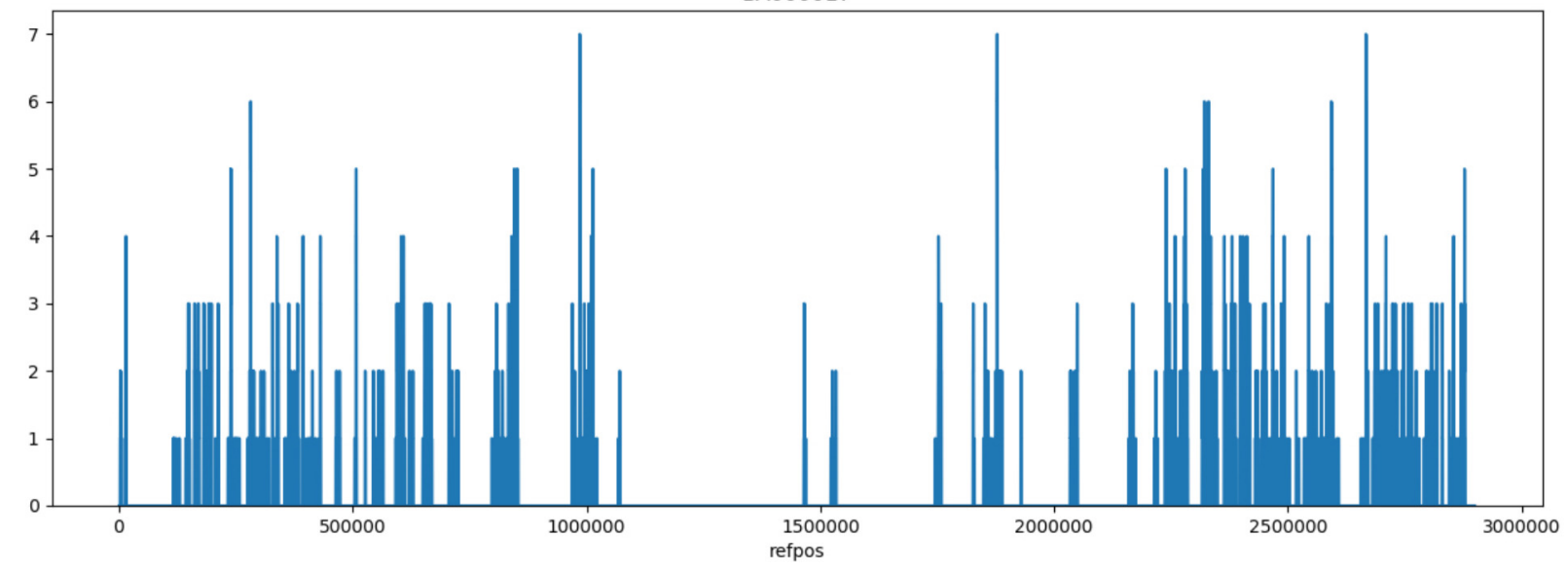

FIGURE 1 | Genomic distribution of SNPS. The position of the 761 identified SNPs were plotted on the Mu50 chromosome using a sliding window of 1,000 bp. SNPs were distributed throughout regions included in the analysis and did not indicate any locations for mutational hotspots. Regions lacking SNP predictions are associated with locations of mobile genetic elements and repeat regions that were excluded from the SNP discovery and encode elements such as the SCCmec element and the $\beta$-hemolysin converting bacteriophage.

single production system or share a common genetic source, with exposure of pigs to LA-MRSA ST5 early in the production system (farrowing unit) and disseminated throughout the later stages as pigs are moved (finishing barns). High resolution profiling provides important phylogenetic signals for strain attribution (Eppinger et al., 2014; Rusconi et al., 2016). In this case, isolates from humans with short-term contact with swine farms could be traced back to a specific farm or production system. For example, isolates ISU886-ISU889 were traced to Farm 24, through SNP analysis. These isolates possessed the unique pattern of SNPs present on that farm or within that production system (Clade IIb, IIc, and IId), indicating exposure to the farm harboring that specific clone. The remaining isolates in Clade II include the isolates from humans with long-term swine contact and UCSF14436. Isolates from humans with long term swine contact did not cluster with isolates from the tested farms (Figure 2). This is consistent with the source of these isolates as it was unlikely the swine veterinarians sampled had contact with the specific farms sourcing the other swine associated isolates in this study. Though these isolates were distinct from the isolates obtained from swine, swine facilities, and humans with short term swine contact, they clustered together within Clade II and were representative of the livestock associated ST5 genotype. A Maximum-likelihood SNP tree was additionally constructed and revealed identical phylogenetic relationship between the MRSA ST5 isolates from humans with no swine contact and the swine-associated LAMRSA ST5 isolates (Supplementary Figure S1).

\section{Mobile Genetic Element Analysis}

The draft genomes of both LA-MRSA ST5 isolates and clinical MRSA ST5 isolates from humans with no swine contact were evaluated for MGEs. LA-MRSA ST5 isolates could also be distinguished from clinical MRSA ST5 isolates by the AMR genes and virulence factors they harbored (Figure 3). For LAMRSA ST5 isolates, the SCCmec elements were of type III,
IV, or untypable. Alternatively, clinical MRSA ST5 isolates harbored predominantly type II SCCmec elements and two type IV elements. Evaluation of the AMR genes revealed the primary macrolide resistance gene was ermA in clinical MRSA ST5 isolates, while LA-MRSA ST5 isolates harbored ermC. Additionally, tetracycline resistance genes were found exclusively in LA-MRSA ST5 isolates. Virulence factors harbored by the subsets were also different, with the majority $(65 / 72,90.3 \%)$ of clinical MRSA ST5 isolates harboring innate immune evasion genes within the $\beta$-hemolysin converting bacteriophage and none of the LA-MRSA ST5 isolates harboring these genes. This analysis revealed that MGE were not commonly shared between the two subsets of isolates.

\section{DISCUSSION}

Methicillin resistant $S$. aureus in livestock species was first identified in the 1970s (Devriese et al., 1972); however, discovery of a high prevalence of MRSA ST398 in swine in 2005, and subsequently in other livestock, brought debate over its public health implications to the forefront (Voss et al., 2005). Previous studies investigating LA-MRSA ST398 demonstrated these isolates have adapted to livestock hosts. This was represented by the absence of human specific virulence factors, such as the $\beta$-hemolysin converting bacteriophage (Price et al., 2012), and reduced adherence and transmission among humans (Graveland et al., 2011; Uhlemann et al., 2012). LA-MRSA ST9 isolates, similar to ST398, are thought to be livestock adapted, and clinical cases appear to be rare and attributed to animal contact (Cuny et al., 2015). While the ST5 lineage in humans is globally distributed and successful in both the hospital and community settings (Monecke et al., 2011), there are currently no reports of MRSA ST5 related disease occurring due to contact with livestock species; however, the genetic potential for swine isolates 


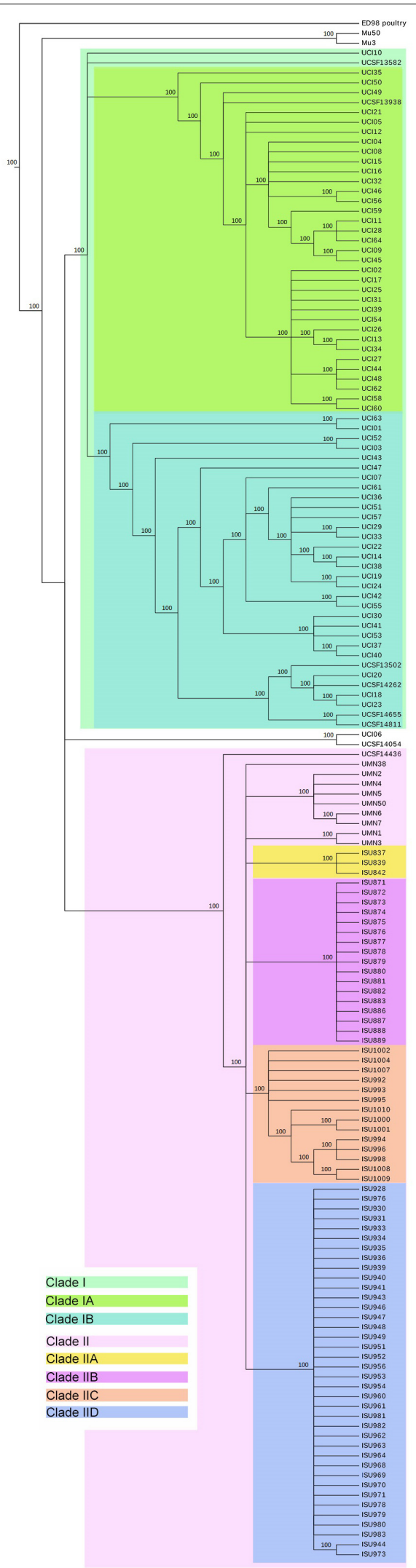

FIGURE 2 | Continued
FIGURE 2 | Maximum parsimony SNP tree of ST5 MRSA isolates. Comparison of 156 genomes, including 153 MRSA ST5 isolates, Mu3, ED98, and Mu50, yielded a total of 764 SNPs, of which 247 were parsimony informative. The tree shown is a majority-consensus tree of 4,440 equally parsimonious trees with a consistency index of 0.9428 . Trees were recovered using a heuristic search in Paup 4.0b10 (Wilgenbusch and Swofford, 2003). This tree is broken into clades, with Clade I representing clinical MRSA ST5 isolates from humans with no swine contact and Clade II representing LA-MRSA ST5 isolates. Clade lla-d are subsets of LA-MRSA ST5 isolates and each subclade represents an individual farm or production system.

to adhere, invade, and cause disease in humans has not been investigated.

Here, we examined the phylogenetic relatedness applying high resolution core genome SNP profiling strategies on MRSA ST5 isolates from swine associated sources and humans with no swine contact to determine if distinct subpopulations of ST5 isolates exist within different host populations, which may provide evidence of host adaption. Similar analyses have been conducted for the ST398 lineage, where researchers identified that the studied LA-MRSA ST398 isolates were within the same clade and that clade appeared to have evolved from an ancestral human methicillin susceptible S. aureus (MSSA) ST398 clade (Price et al., 2012).

In this study, isolates from humans with no swine contact clustered distinctly from swine associated LA-MRSA ST5 isolates. All swine associated LA-MRSA ST5 isolates were contained within Clade II, while all but one MRSA ST5 from humans with no swine contact belonged to Clade I. The MGE complement of this clinical MRSA ST5 isolate (UCSF14436) suggests that it is likely a CA-MRSA isolate. CA-MRSA isolates tend to be genetically distinct from HA-MRSA isolates (Stefani et al., 2012), which may explain why UCSF 14436 is found within the LA-MRSA clade. The separation of LA-MRSA ST5 isolates from clinical MRSA ST5 isolates based on their core genome relatedness supports our previous work, which indicated that swine associated LA-MRSA ST5 isolates harbor different MGEs containing virulence and resistance genes than MRSA ST5 from humans with no swine contact (Hau et al., 2015, 2017g; Hau, 2017). The data reported here further demonstrate the phylogenetic distinction of isolates into different groups and may reflect the adaptation of LA-MRSA ST5 isolates to colonization of swine. This distinction is based on focusing on MGEs harboring virulence factors and/or AMR and not on the full complement of all MGEs. This focus was warranted to begin evaluating the potential of LA-MRSA ST5 isolates to serve as a source for human infections or as a source for MGEs harboring virulence factors or AMR based on the isolates included in this study. Furthermore, similar to LA-MRSA ST398, evaluation of known virulence genes provides evidence that swine associated LA-MRSA ST5 may be less capable of causing disease in humans (Figure 3) (Hau et al., 2015). The $d N / d S$ ratio observed within the core genome of the isolates was 2.3 , which could potentially indicate that these strains recently evolved or underwent initial stages of diversification (Castillo-Ramirez et al., 2011).

Our results indicate frequent introduction of MRSA ST5 onto swine farms is unlikely and dominant clones of MRSA 


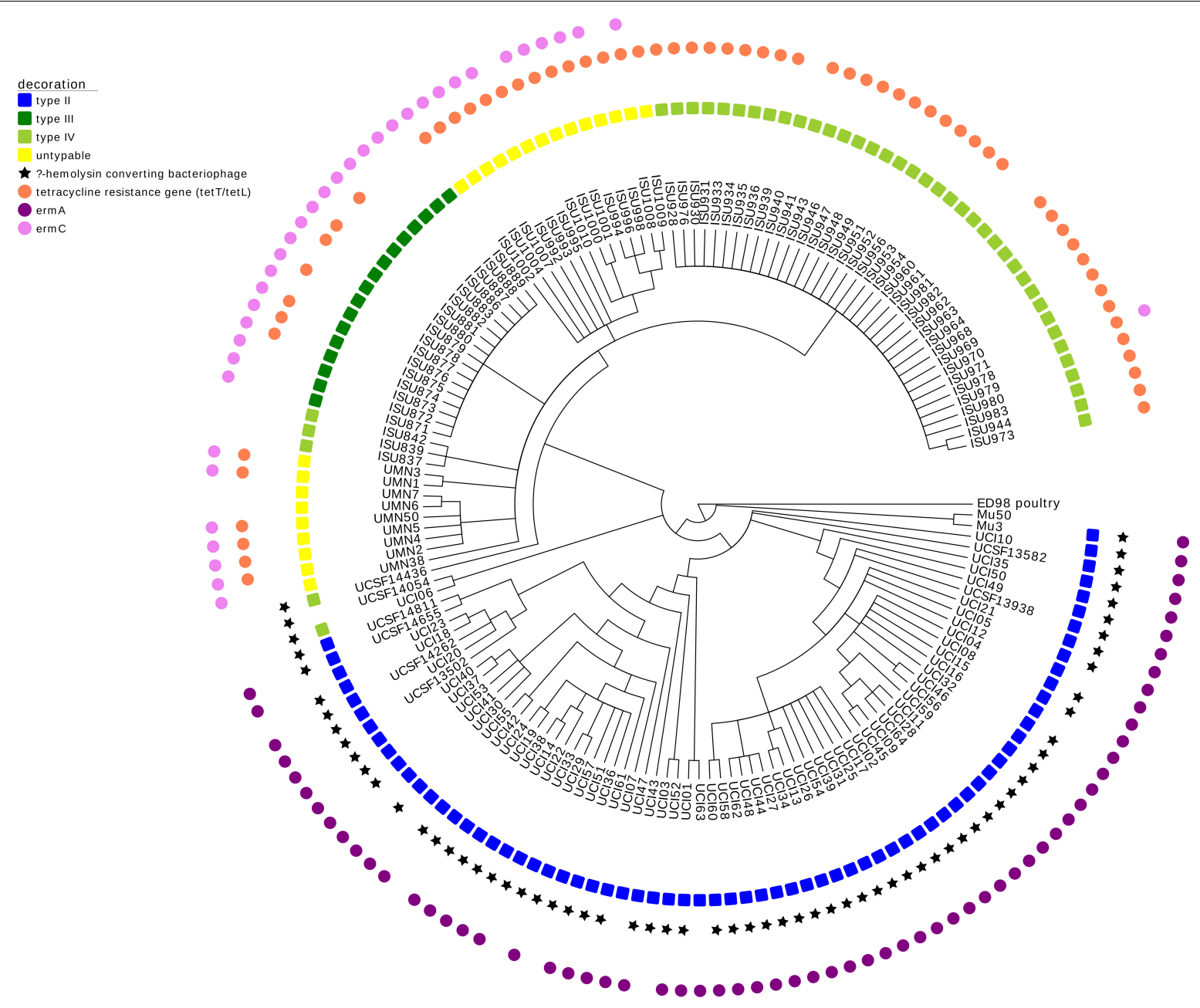

FIGURE 3 | Maximum parsimony SNP tree of ST5 MRSA isolates with MGE analysis. The SNP tree developed for Figure $\mathbf{2}$ was decorated using Evolview (He et al., 2016). The tree shows the MGE complement of these isolates, specifically describing the SCCmec element, the $\beta$-hemolysin converting bacteriophage (harboring virulence factors involved in innate immune evasion), and antimicrobial resistance genes involved in tetracycline resistance (tetT/tetL) and macrolide resistance (ermA and ermC).

ST5 circulated within each farm at the time of sampling. A high degree of genetic relatedness was identified among swineassociated isolates. As expected, the population of isolates found on individual farms were dominated by clonal populations. This indicates either a single introduction of LA-MRSA ST5 onto a farm or dominance of a single variant that precludes colonization with alternative isolates introduced by human caretakers. However, multiple $S$. aureus lineages and spa types often occur simultaneously on swine farms and in individual pigs (Linhares et al., 2015), so the diversity of $S$. aureus in these animals may be underrepresented when looking specifically at MRSA ST5 isolates. This clonality was not present in isolates from humans with no swine contact, where genome plasticity was greater and individual isolates showed more diversity than that observed among LA-MRSA ST5 isolates.

While the evidence here suggests the populations of LA-MRSA ST5 and MRSA ST5 from humans with no swine contact are distinct, the isolates used in this analysis were sourced from geographically separated regions to ensure the clinical isolates were from patients with no swine contact. This design was utilized to build a solid reference point for comparing MRSA ST5 isolates from clinical and agricultural sources; however, because of the unlikelihood of these populations mingling, the SNPs identified can be attributed to either the geographic separation or the host species of the isolates. Future studies are warranted that include clinical MRSA ST5 isolates obtained from the hospital and community setting in areas of dense swine production, enabling further elucidation of the potential of LA-MRSA ST5 isolates to serve as a source for human infections or as a source for MGEs harboring virulence factors or AMR. As evidenced in this study, WGS followed by high resolution SNP profiling is a powerful molecular genomic epidemiology approach to gain insights into the population structure of LA-, CA-, and HA-MRSA and can provide important phylogenetic indicators for strain attribution. 


\section{AUTHOR CONTRIBUTIONS}

SH, AA-G, BR, JH, PD, TF, ME, and TN conceived and designed the experiments, contributed reagents, materials, and analysis tools, and wrote the paper. SH, AA-G, and BR performed the experiments. SH, AA-G, BR, ME, and TN analyzed the data. All authors gave approval of the final version to be published and agreed to be accountable for all aspects of the work.

\section{FUNDING}

This research was conducted in part with funding from the National Pork Board and the United States Department of Agriculture, Agriculture Research Service.

\section{ACKNOWLEDGMENTS}

The authors would like to thank Dr. Susan Huang from the University of California, Irvine, School of Medicine and Dr. Binh Diep from the Department of Medicine, University of California, San Francisco for providing the clinical strains used in this study. Mention of trade names or commercial products in this

\section{REFERENCES}

Altschul, S. F., Gish, W., Miller, W., Myers, E. W., and Lipman, D. J. (1990). Basic local alignment search tool. J. Mol. Biol. 215, 403-410. doi: 10.1016/S00222836(05)80360-2

Andreoletti, O., Budka, H., Buncic, S., Colin, P., Collins, J. D., De Koeijer, A., et al. (2009). Scientific opinion of the panel on biological hazards on a request from the European commission on assessment of the public health significance of meticillin resistant Staphylococcus aureus (MRSA) in animals and foods. EFSA J. 993, 1-73.

Bal, A. M., Coombs, G. W., Holden, M. T., Lindsay, J. A., Nimmo, G. R., Tattevin, P., et al. (2016). Genomic insights into the emergence and spread of international clones of healthcare-, community- and livestockassociated meticillin-resistant Staphylococcus aureus: blurring of the traditional definitions. J Glob Antimicrob Resist 6, 95-101. doi: 10.1016/j.jgar.2016. 04.004

Castillo-Ramirez, S., Harris, S. R., Holden, M. T., He, M., Parkhill, J., Bentley, S. D., et al. (2011). The impact of recombination on $\mathrm{dN} / \mathrm{dS}$ within recently emerged bacterial clones. PLoS Pathog. 7:e1002129. doi: 10.1371/journal.ppat.10 02129

Cuny, C., Wieler, L. H., and Witte, W. (2015). Livestock-Associated MRSA: the Impact on Humans. Antibiotics 4, 521-543. doi: 10.3390/antibiotics4040521

Delcher, A. L., Salzberg, S. L., and Phillippy, A. M. (2003). Using MUMmer to identify similar regions in large sequence sets. Curr. Protoc. Bioinformatics 10.3.1-10.3.18. doi: 10.1002/0471250953.bi1003s00

DeLeo, F. R., and Chambers, H. F. (2009). Reemergence of antibiotic-resistant Staphylococcus aureus in the genomics era. J. Clin. Invest. 119, 2464-2474. doi: $10.1172 /$ JCI38226

Devriese, L. A., Van Damme, L. R., and Fameree, L. (1972). Methicillin (cloxacillin)-resistant Staphylococcus aureus strains isolated from bovine mastitis cases. Zentralbl Veterinarmed B 19, 598-605. doi: 10.1111/j.1439-0450. 1972.tb00439.x

Eppinger, M., Mammel, M. K., Leclerc, J. E., Ravel, J., and Cebula, T. A. (2011). Genomic anatomy of Escherichia coli O157:H7 outbreaks. Proc. Natl. Acad. Sci. U.S.A. 108, 20142-20147. doi: 10.1073/pnas.1107176108

Eppinger, M., Pearson, T., Koenig, S. S., Pearson, O., Hicks, N., Agrawal, S., et al. (2014). Genomic epidemiology of the Haitian cholera outbreak: a single introduction followed by rapid, extensive, and continued spread characterized the onset of the epidemic. mBio 5:e01721. doi: 10.1128/mBio.01721-14 article is solely for the purpose of providing specific information and does not imply recommendation or endorsement by the United States Department of Agriculture. USDA is an equal opportunity provider and employer. This research was originally published as a component of the Ph.D. dissertation of SH (Hau, 2017) and is available online through Iowa State University's Digital Repository.

\section{SUPPLEMENTARY MATERIAL}

The Supplementary Material for this article can be found online at: https://www.frontiersin.org/articles/10.3389/fmicb. 2018.02078/full\#supplementary-material

FIGURE S1 | Maximum-likelihood (ML) SNP tree of ST5 MRSA isolates obtained by RAxML. A Maximum-likelihood SNP tree was constructed from the 764 SNPS identified from the comparison of 156 genomes, including 153 MRSA ST5 isolates, Mu3, ED98, and Mu50.

TABLE S1 | Genome sequencing statistics and MGE composition for all isolates used in study.

TABLE S2 | General features linked to identified SNPs.

TABLE S3 | Specific genomic features linked to identified SNPs.

Fitzgerald, J. R. (2012). Human origin for livestock-associated methicillin-resistant Staphylococcus aureus. mBio 3:e0082-12. doi: 10.1128/mBio.00082-12

Frana, T. S., Beahm, A. R., Hanson, B. M., Kinyon, J. M., Layman, L. L., Karriker, L. A., et al. (2013). Isolation and characterization of methicillin-resistant Staphylococcus aureus from pork farms and visiting veterinary students. PLoS One 8:e53738. doi: 10.1371/journal.pone.0053738

Garrison, E., and Marth, G. (2012). Haplotype-based variant detection from short-read sequencing. arXiv:1207.3907v2 [Preprint].

Giardine, B., Riemer, C., Hardison, R. C., Burhans, R., Elnitski, L., Shah, P., et al. (2005). Galaxy: a platform for interactive large-scale genome analysis. Genome Res. 15, 1451-1455. doi: 10.1101/gr.4086505

Goecks, J., Nekrutenko, A., Taylor, J., and Galaxy, T. (2010). Galaxy: a comprehensive approach for supporting accessible, reproducible, and transparent computational research in the life sciences. Genome Biol. 11:R86. doi: 10.1186/gb-2010-11-8-r86

Graveland, H., Duim, B., Van Duijkeren, E., Heederik, D., and Wagenaar, J. A. (2011). Livestock-associated methicillin-resistant Staphylococcus aureus in animals and humans. Int. J. Med. Microbiol. 301, 630-634. doi: 10.1016/j.ijmm. 2011.09.004

Hau, S. J. (2017). Genetic and Phenotypic Characterization of Livestock Associated Methicillin Resistant Staphylococcus aureus Sequence Type (ST) 5 in Comparison with Clinical ST5 Isolates from Humans. Ph.D., Iowa State University, Ames, IA. doi: $10.31274 /$ etd-180810-5772

Hau, S. J., Bayles, D. O., Alt, D. P., Davies, P. R., Haan, J. S., and Nicholson, T. L. (2017a). Draft genome sequences of nine livestock-associated methicillinresistant Staphylococcus aureus sequence type 5 isolates from humans with longterm swine contact. Genome Announc 5:e01079-17. doi: 10.1128/genomeA. 01079-17

Hau, S. J., Bayles, D. O., Alt, D. P., Frana, T. S., and Nicholson, T. L. (2017b). Draft genome sequences of 63 swine-associated methicillin-resistant Staphylococcus aureus sequence type 5 isolates from the United States. Genome Announc. 5:e01081-17. doi: 10.1128/genomeA.01081-17

Hau, S. J., Bayles, D. O., Alt, D. P., Frana, T. S., and Nicholson, T. L. (2017c). Draft genome sequences of nine livestock-associated methicillinresistant Staphylococcus aureus sequence type 5 isolates obtained from humans after short-term swine contact. Genome Announc. 5:e01080-17. doi: 10.1128/ genomeA.01080-17

Hau, S. J., Bayles, D. O., Alt, D. P., and Nicholson, T. L. (2017d). Draft Genome Sequences of 14 Staphylococcus aureus sequence type 5 isolates from California, 
USA. Microbiol. Resour. Announc. 5:e00098-17. doi: 10.1128/genomeA.000 98-17

Hau, S. J., Bayles, D. O., Alt, D. P., and Nicholson, T. L. (2017e). Draft genome sequences of 50 methicillin-resistant Staphylococcus aureus sequence type 5 isolates obtained from a U.S. hospital. Genome Announc. 5:e01083-17. doi: 10.1128/genomeA.01083-17

Hau, S. J., Bayles, D. O., Alt, D. P., and Nicholson, T. L. (2017f). Draft genome sequences of one methicillin-sensitive and seven methicillin-resistant Staphylococcus aureus sequence type 5 isolates obtained in California. Genome Announc. 5:e01084-17. doi: 10.1128/genomeA.01084-17

Hau, S. J., Frana, T., Sun, J., Davies, P. R., and Nicholson, T. L. (2017g). Zinc resistance within swine associated methicillin resistant Staphylococcus aureus (MRSA) isolates in the USA is associated with MLST lineage. Appl Environ Microbiol 83:e00756-17. doi: 10.1128/AEM.00756-17

Hau, S. J., Sun, J., Davies, P. R., Frana, T. S., and Nicholson, T. L. (2015). Comparative prevalence of immune evasion complex genes associated with beta-hemolysin converting bacteriophages in MRSA ST5 isolates from swine, swine facilities, humans with swine contact, and humans with no swine contact. PLoS One 10:e0142832. doi: 10.1371/journal.pone.0142832

He, Z., Zhang, H., Gao, S., Lercher, M. J., Chen, W. H., and Hu, S. (2016). Evolview v2: an online visualization and management tool for customized and annotated phylogenetic trees. Nucleic Acids Res. 44, W236-W241. doi: 10.1093/ nar/gkw370

Hiramatsu, K., Aritaka, N., Hanaki, H., Kawasaki, S., Hosoda, Y., Hori, S., et al. (1997). Dissemination in Japanese hospitals of strains of Staphylococcus aureus heterogeneously resistant to vancomycin. Lancet 350, 1670-1673. doi: 10.1016/ S0140-6736(97)07324-8

Hudson, L. O., Murphy, C. R., Spratt, B. G., Enright, M. C., Elkins, K., Nguyen, C., et al. (2013). Diversity of methicillin-resistant Staphylococcus aureus (MRSA) strains isolated from inpatients of 30 hospitals in Orange County, California. PLoS One 8:e62117. doi: 10.1371/journal.pone.0062117

Kearse, M., Moir, R., Wilson, A., Stones-Havas, S., Cheung, M., Sturrock, S., et al. (2012). Geneious Basic: an integrated and extendable desktop software platform for the organization and analysis of sequence data. Bioinformatics 28 , 1647-1649. doi: 10.1093/bioinformatics/bts199

Klevens, R. M., Morrison, M. A., Nadle, J., Petit, S., Gershman, K., Ray, S., et al. (2007). Invasive methicillin-resistant Staphylococcus aureus infections in the United States. JAMA 298, 1763-1771. doi: 10.1001/jama.298.15.1763

Langmead, B., and Salzberg, S. L. (2012). Fast gapped-read alignment with Bowtie 2. Nat. Methods 9, 357-359. doi: 10.1038/nmeth.1923

Linhares, L. L., Yang, M., Sreevatsan, S., Munoz-Zanzi, C. A., Torremorell, M., and Davies, P. R. (2015). The effect of anatomic site and age on detection of Staphylococcus aureus in pigs. J. Vet. Diagn. Invest. 27, 55-60. doi: 10.1177/ 1040638714559598

Lowder, B. V., Guinane, C. M., Ben Zakour, N. L., Weinert, L. A., ConwayMorris, A., Cartwright, R. A., et al. (2009). Recent human-to-poultry host jump, adaptation, and pandemic spread of Staphylococcus aureus. Proc. Natl. Acad. Sci. U.S.A. 106, 19545-19550. doi: 10.1073/pnas.0909285106

Maddison, W. P., and Maddison, D. R. (2017). ). Mesquite: a Modular System for Evolutionary Analysis Version3.2.

Molla, B., Byrne, M., Abley, M., Mathews, J., Jackson, C. R., Fedorka-Cray, P., et al. (2012). Epidemiology and genotypic characteristics of methicillinresistant Staphylococcus aureus strains of porcine origin. J. Clin. Microbiol. 50, 3687-3693. doi: 10.1128/JCM.01971-12

Monecke, S., Coombs, G., Shore, A. C., Coleman, D. C., Akpaka, P., Borg, M., et al. (2011). A field guide to pandemic, epidemic and sporadic clones of methicillinresistant Staphylococcus aureus. PLoS One 6:e17936. doi: 10.1371/journal.pone. 0017936

Myers, G. S., Mathews, S. A., Eppinger, M., Mitchell, C., O’brien, K. K., White, O. R., et al. (2009). Evidence that human Chlamydia pneumoniae was zoonotically acquired. J Bacteriol 191, 7225-7233. doi: 10.1128/JB.00746-09

Pantosti, A. (2012). Methicillin-resistant Staphylococcus aureus associated with animals and its relevance to human health. Front. Microbiol. 3:127. doi: 10.3389/ fmicb.2012.00127

Price, L. B., Stegger, M., Hasman, H., Aziz, M., Larsen, J., Andersen, P. S., et al. (2012). Staphylococcus aureus CC398: host adaptation and emergence of methicillin resistance in livestock. mBio 3:e00520-12. doi:10.1128/mBio.003 05-11
Rusconi, B., Sanjar, F., Koenig, S. S., Mammel, M. K., Tarr, P. I., and Eppinger, M. (2016). Whole genome sequencing for genomics-guided investigations of Escherichia coli O157:H7 outbreaks. Front. Microbiol. 7:985. doi: 10.3389/fmicb. 2016.00985

Sahibzada, S., Abraham, S., Coombs, G. W., Pang, S., Hernandez-Jover, M., Jordan, D., et al. (2017). Transmission of highly virulent communityassociated MRSA ST93 and livestock-associated MRSA ST398 between humans and pigs in Australia. Sci. Rep. 7:5273. doi: 10.1038/s41598-017-04 789-0

Schijffelen, M. J., Boel, C. H., Van Strijp, J. A., and Fluit, A. C. (2010). Whole genome analysis of a livestock-associated methicillin-resistant Staphylococcus aureus ST398 isolate from a case of human endocarditis. BMC Genomics 11:376. doi: 10.1186/1471-2164-11-376

Siguier, P., Perochon, J., Lestrade, L., Mahillon, J., and Chandler, M. (2006). ISfinder: the reference centre for bacterial insertion sequences. Nucleic Acids Res. 34, D32-D36. doi: 10.1093/nar/gkj014

Spoor, L. E., Mcadam, P. R., Weinert, L. A., Rambaut, A., Hasman, H., Aarestrup, F. M., et al. (2013). Livestock origin for a human pandemic clone of communityassociated methicillin-resistant Staphylococcus aureus. mBio 4:e00356-13. doi: 10.1128/mBio.00356-13

Stamatakis, A. (2014). RAxML version 8: a tool for phylogenetic analysis and post-analysis of large phylogenies. Bioinformatics 30, 1312-1313. doi: 10.1093/ bioinformatics/btu033

Stefani, S., Chung, D. R., Lindsay, J. A., Friedrich, A. W., Kearns, A. M., Westh, H., et al. (2012). Meticillin-resistant Staphylococcus aureus (MRSA): global epidemiology and harmonisation of typing methods. Int. J. Antimicrob. Agents 39, 273-282. doi: 10.1016/j.ijantimicag.2011.09.030

Sun, J., Yang, M., Sreevatsan, S., Bender, J. B., Singer, R. S., Knutson, T. P., et al. (2017). Longitudinal study of Staphylococcus aureus colonization and infection in a cohort of swine veterinarians in the United States. BMC Infect. Dis. 17:690. doi: 10.1186/s12879-017-2802-1

Sun, J., Yang, M., Sreevatsan, S., and Davies, P. R. (2015). Prevalence and Characterization of Staphylococcus aureus in Growing Pigs in the USA. PLoS One 10:e0143670. doi: 10.1371/journal.pone.0143670

Uhlemann, A. C., Porcella, S. F., Trivedi, S., Sullivan, S. B., Hafer, C., Kennedy, A. D., et al. (2012). Identification of a highly transmissible animal-independent Staphylococcus aureus ST398 clone with distinct genomic and cell adhesion properties. MBio 3:e00027-12. doi: 10.1128/mBio.00027-12

Voss, A., Loeffen, F., Bakker, J., Klaassen, C., and Wulf, M. (2005). Methicillinresistant Staphylococcus aureus in pig farming. Emerg. Infect. Dis 11, 1965-1966. doi: 10.3201/eid1112.050428

Wagenaar, J. A., Yue, H., Pritchard, J., Broekhuizen-Stins, M., Huijsdens, X., Mevius, D. J., et al. (2009). Unexpected sequence types in livestock associated methicillin-resistant Staphylococcus aureus (MRSA): MRSA ST9 and a single locus variant of ST9 in pig farming in China. Vet Microbiol 139, 405-409. doi: 10.1016/j.vetmic.2009.06.014

Wilgenbusch, J. C., and Swofford, D. (2003). Inferring evolutionary trees with PAUP*. Curr. Protoc. Bioinformatics 6.4.1-6.4.28. doi: 10.1002/0471250953. bi0604s00

Zankari, E., Hasman, H., Cosentino, S., Vestergaard, M., Rasmussen, S., Lund, O., et al. (2012). Identification of acquired antimicrobial resistance genes. $J$ Antimicrob Chemother 67, 2640-2644. doi: 10.1093/jac/dks261

Zhou, Y., Liang, Y., Lynch, K. H., Dennis, J. J., and Wishart, D. S. (2011). PHAST: a fast phage search tool. Nucleic Acids Res. 39, W347-W352. doi: 10.1093/nar/ gkr485

Conflict of Interest Statement: The authors acknowledge receiving funding from the National Pork Board, however, declare that the funding sources in no way impacted experimental design, data analysis, manuscript preparation, or the decision to publish.

Copyright (c) 2018 Hau, Allué-Guardia, Rusconi, Haan, Davies, Frana, Eppinger and Nicholson. This is an open-access article distributed under the terms of the Creative Commons Attribution License (CC BY). The use, distribution or reproduction in other forums is permitted, provided the original author(s) and the copyright owner(s) are credited and that the original publication in this journal is cited, in accordance with accepted academic practice. No use, distribution or reproduction is permitted which does not comply with these terms. 